\title{
Non-Linear Inner Structure of Topological Vector Spaces
}

\author{
Francisco Javier García-Pacheco ${ }^{1, *,+} \oplus$, Soledad Moreno-Pulido ${ }^{1,+} \oplus$, Enrique Naranjo-Guerra ${ }^{1,+}$ \\ and Alberto Sánchez-Alzola ${ }^{2,+}$ (D) \\ 1 Department of Mathematics, College of Engineering, University of Cadiz, 11519 Puerto Real, CA, Spain; \\ soledad.moreno@uca.es (S.M.-P.); enrique.naranjo@uca.es (E.N.-G.) \\ 2 Department of Statistics and Operation Research, College of Engineering, University of Cadiz, \\ 11519 Puerto Real (CA), Spain; alberto.sanchez@uca.es \\ * Correspondence: garcia.pacheco@uca.es \\ + These authors contributed equally to this work.
}

Citation: García-Pacheco, F.J.; Moreno-Pulido, S.; Naranjo-Guerra, E.; Sánchez-Alzola, A. Non-Linear Inner Structure of Topological Vector Spaces. Mathematics 2021, 9, 466. https://doi.org/math9050466

Academic Editor: Andrea Scapellato

Received: 21 December 2020

Accepted: 20 February 2021

Published: 25 February 2021

Publisher's Note: MDPI stays neutral with regard to jurisdictional claims in published maps and institutional affiliations.

Copyright: (C) 2021 by the authors. Licensee MDPI, Basel, Switzerland. This article is an open access article distributed under the terms and conditions of the Creative Commons Attribution (CC BY) license (https:/ / creativecommons.org/licenses/by/ $4.0 /)$.

\begin{abstract}
Inner structure appeared in the literature of topological vector spaces as a tool to characterize the extremal structure of convex sets. For instance, in recent years, inner structure has been used to provide a solution to The Faceless Problem and to characterize the finest locally convex vector topology on a real vector space. This manuscript goes one step further by settling the bases for studying the inner structure of non-convex sets. In first place, we observe that the well behaviour of the extremal structure of convex sets with respect to the inner structure does not transport to non-convex sets in the following sense: it has been already proved that if a face of a convex set intersects the inner points, then the face is the whole convex set; however, in the non-convex setting, we find an example of a non-convex set with a proper extremal subset that intersects the inner points. On the opposite, we prove that if a extremal subset of a non-necessarily convex set intersects the affine internal points, then the extremal subset coincides with the whole set. On the other hand, it was proved in the inner structure literature that isomorphisms of vector spaces and translations preserve the sets of inner points and outer points. In this manuscript, we show that in general, affine maps and convex maps do not preserve inner points. Finally, by making use of the inner structure, we find a simple proof of the fact that a convex and absorbing set is a neighborhood of 0 in the finest locally convex vector topology. In fact, we show that in a convex set with internal points, the subset of its inner points coincides with the subset of its internal points, which also coincides with its interior with respect to the finest locally convex vector topology.
\end{abstract}

Keywords: topological vector space; internal point; convex; balanced; absorbing; inner structure

MSC: 46A03; 46A55; 46H25

\section{Introduction}

The main goal of this work is to entail a study of inner structure in non-necessarily convex sets. As we have mentioned above, inner structure is a relatively new theory that appeared for the first time in [1] and that has been strongly applied to study the topological and extremal properties of convex sets [2,3]. For instance, in [3], inner structure has been used to characterize the finest locally convex vector topology on a real vector space. In [2], inner structure was applied to solve The Faceless Problem. The origin and motivation of inner structure dates back to $[4,5]$ under the concept of internal point in a convex setting, which we will define and use later on.

This manuscript is divided into eight sections. The first section is the introduction, where we present the state of the art, origins, motivations and objectives of this work. The second section is on the preliminary definitions, i.e., the most basic concepts involved in this paper are presented (the most advanced concepts and tools involved will be recalled alongside this manuscript). In the third section, we provide in Proposition 2 a characteri- 
zation of convex sets and starlike sets in terms of the set $\operatorname{shl}(M, m)$. In the fourth section, we prove that the inner points are, in general, not preserved by affine maps nor by convex maps (see Theorems 1-4). In the fifth section, we revisit the finest locally convex vector topology [6,7] from the point of view provided by the inner points. In particular, we show in Lemma 5 that in a convex set with internal points, the subset of its inner points coincides with the subset of its internal points, which also coincides with its interior with respect to the finest locally convex vector topology (see [4,8-10] for a wide perspective on Hausdorff locally convex topological vector spaces and [11-13] for a wider perspective on topological modules). As a consequence, we provide a new proof of the fact that every convex absorbing subset of a vector space is a neighborhood of 0 in the finest locally convex vector topology (see Theorem 6). In the sixth section, we characterize the linearly closed sets (see Corollary 3) and then we construct non-trivial examples of non-convex, balanced, absorbing and linearly open sets (see Theorems 7-9), which are in fact candidate not to be neighborhoods of zero in the finest locally convex vector topology (for this we strongly rely on [14,15], where plenty of examples of balanced an absorbing sets with empty interior were provided for any Hausdorff locally convex topological vector space of dimension greater than or equal to 2). In the seventh section, in Example 3, we provide an example of a non-convex set with a proper extremal subset that intersects the inner points (in [2] it was shown that a proper convex extremal subset of a convex set never intersects the inner points). We also prove in the seventh section that if a extremal subset of a non-necessarily convex set intersects the affine internal points, then the extremal subset coincides with the whole set (Theorem 10). Finally, in the eighth and last section, we prove that if a set is linearly closed, then so are its convex components (see Theorem 13).

\section{Preliminaries}

All the vector spaces considered throughout this manuscript will be over the reals. A closed segment in a vector space is a set of the form $[x, y]:=\{t x+(1-t) y: t \in[0,1]\}$, an open segment is a set of the form $(x, y):=\{t x+(1-t) y: t \in(0,1)\}$, and a semi-open (or semi-closed) segment has the form $[x, y):=\{t y+(1-t) x: t \in[0,1)\}$. We will refer to closed, open, semi-closed or semi-open segments simply by segments. A segment is said to be non-trivial when the extremes are different.

A line passing through $x \neq y$ is a set of the form $(-\infty ; x, y ; \infty):=\{t x+(1-t) y$ : $t \in \mathbb{R}\}$. A closed ray starting at $x$ and passing through $y \neq x$ is a set of the form $[x, y ; \infty):=\{(1-t) x+t y: t \in[0,+\infty)\}$ and an open ray starting at $x$ and passing through $y \neq x$ is a set of the form $(x, y ; \infty):=\{(1-t) x+t y: t \in(0,+\infty)\}$. We will refer to closed rays and open rays simply by rays.

Given a subset $M$ of a vector space $X$, the set $\operatorname{shl}(M)$, consisting of all the non-trivial segments, the rays and the lines of $X$ contained in $M$, can be partially ordered by the inclusion. It is not difficult to check, by relying on Zorn's Lemma, that there are always maximal elements in $\operatorname{shl}(M)$. In fact, every element of $\operatorname{shl}(M)$ is always contained in a maximal element.

We also define the set $\operatorname{shl}(M, S):=\{T \in \operatorname{shl}(M): S \subseteq T\}$, where $S$ is a subset of $M$. It is trivial that every maximal element of $\operatorname{shl}(M, S)$ is also a maximal element of $\operatorname{shl}(M)$. For simplicity, $\operatorname{shl}(M, m):=\operatorname{shl}(M,\{m\})$ for every $m \in M$. Please note that $\operatorname{shl}(M, \varnothing)=\operatorname{shl}(M)$ so whenever we write $\operatorname{shl}(M, S)$ we will be assuming that $S \neq \varnothing$.

The following definition can be found in [1] (Definition 1.2).

Definition 1. Let $X$ be a vector space, $M$ a subset of $X$ and $x \in X$.

1. We will say that $x$ is an inner point of $M$ if $x \in M, \operatorname{shl}(M, x) \neq \varnothing$ and every maximal element of $\operatorname{shl}(M, x)$ has $x$ in its interior. The set of inner points of $M$ will be denoted by $\operatorname{inn}(M)$.

2. We will say that $x$ is an outer point of $M$ if either $x$ is an extreme of some maximal element of $\operatorname{shl}(M)$ or $x \in M$ with $\operatorname{shl}(M, x)=\varnothing$. The set of outer points of $M$ will be denoted by $\operatorname{out}(M)$. 
Notice that $\operatorname{inn}(M) \cap \operatorname{out}(M)=\varnothing$ and thus $\{\operatorname{inn}(M), \operatorname{out}(M) \cap M\}$ is a partition of $M$. The linear closure of a set $M$ is defined as $\operatorname{lcl}(M):=\operatorname{inn}(M) \cup \operatorname{out}(M)$.

The following remark is very useful when it comes to showing that a certain point is an inner point of a convex set.

Remark 1. Let $X$ be a vector space and $M$ a non-singleton convex subset of $X$. A point $m \in M$ is an inner point of $M$ if and only if for every $n \in M \backslash\{m\}$ there exists $t<0$ such that $(1-t) m+t n \in M$.

\section{Geometric Forms Determined by $\operatorname{shl}(M, m)$}

The following proposition, which is a refinement of [1] (Proposition 1.1), describes explicitly the maximal elements of $\operatorname{shl}(M)$. In order for that, we recall the reader that a line in a vector space can be canonically endowed with a topology that makes it homeomorphic to $\mathbb{R}$. Since the connected subsets of $\mathbb{R}$ are exactly the intervals, the connected subsets of a line are exactly its segments, its rays and itself.

Proposition 1. Let $X$ be a vector space, $M$ a non-singleton non-empty subset of $X$ and $T$ a non-empty subset of $M$. The following conditions are equivalent:

1. $T$ is a maximal element of $\operatorname{shl}(M)$.

2. There exists a line $L$ of $X$ such that $T$ is a non-singleton connected component of $L \cap M$.

Proof. Suppose first that $T$ is a maximal element of $\operatorname{shl}(M)$. By definition, $T$ is not a singleton. Let $L$ be the line of $X$ containing $T$. Denote by $S$ to the connected component of $L \cap M$ containing $T$. Since $T$ is not a singleton, then $S$ is not a singleton either. Please note that $S$ is convex and thus $S \in \operatorname{shl}(M, T)$, so by maximality $T=S$. Conversely, suppose that there exists a line $L$ of $X$ such that $T$ is a non-singleton connected component of $L \cap M$. Since $T$ is convex, we conclude that $T \in \operatorname{shl}(M)$. Let $S \in \operatorname{shl}(M, T)$. Since $T$ has more than one point and $T \subseteq S$, we have that $S \subseteq L$, therefore $T \subseteq S \subseteq L \cap M$. Since $S$ is connected, we conclude that $T=S$ and thus $T$ is a maximal element of $\operatorname{shl}(M)$.

Recall that if $A$ is a set, then the notation $\bigcup A$ stands for $\bigcup_{B \in A} B$. Recall also that a subset $A$ of a vector space $X$ is said to be starlike provided that there exists $a \in A$, called the center of $A$, such that $[a, b] \subseteq A$ for all $b \in A$.

Proposition 2. Let $X$ be a vector space and $M$ a subset of $X$ with at least two points.

1. $M$ is convex if and only if $M=\cup \operatorname{shl}(M, m)$ for all $m \in M$.

2. $M$ is starlike if and only if $M=\bigcup \operatorname{shl}(M, m)$ for some $m \in M$.

\section{Proof.}

1. Assume first that $M$ is convex. Fix an arbitrary $m_{0} \in M$. By definition, $\bigcup \operatorname{shl}\left(M, m_{0}\right) \subseteq$ $M$. We will show that $M \subseteq \cup \operatorname{shl}\left(M, m_{0}\right)$. Take $m_{1} \in M \backslash\left\{m_{0}\right\}$. The convexity of $M$ allows that the non-trivial segment $\left[m_{0}, m_{1}\right] \in \operatorname{shl}\left(M, m_{0}\right)$. As a consequence, $m_{1} \in \bigcup \operatorname{shl}\left(M, m_{0}\right)$. Conversely, assume that $M=\bigcup \operatorname{shl}(M, m)$ for all $m \in M$. Fix two arbitrary elements $m \neq n \in M$. By assumption, $m \in M=\bigcup \operatorname{shl}(M, n)$, therefore there exists an element $T \in \operatorname{shl}(M, n)$ such that $m \in T$. Since $T$ is convex, we have that $[m, n] \subseteq T \subseteq M$.

2. Assume first that $M$ is starlike and let $m_{0} \in M$ its center. By definition, $\bigcup \operatorname{shl}\left(M, m_{0}\right) \subseteq$ $M$. We will show that $M \subseteq \bigcup \operatorname{shl}\left(M, m_{0}\right)$. Take $m_{1} \in M \backslash\left\{m_{0}\right\}$. The starlike character of $M$ allows that the non-trivial segment $\left[m_{0}, m_{1}\right] \in \operatorname{shl}\left(M, m_{0}\right)$. As a consequence, $m_{1} \in \bigcup \operatorname{shl}\left(M, m_{0}\right)$. Conversely, assume that $M=\bigcup \operatorname{shl}(M, m)$ for some $m \in M$. Fix an arbitrary element $n \in M \backslash\{m\}$. By assumption, $n \in M=\bigcup \operatorname{shl}(M, m)$, therefore there exists an element $T \in \operatorname{shl}(M, m)$ such that $n \in T$. Since $T$ is convex, we have that $[m, n] \subseteq T \subseteq M$. 


\section{Inner-Point Preserving Maps}

In [1] (Proposition 1.3) the following proposition was remarked without a proof.

Proposition 3. Let $X$ be a vector space and $M$ a non-empty subset of $X$.

1. $\operatorname{inn}(M+x)=\operatorname{inn}(M)+x$ for all $x \in X$.

2. $\operatorname{out}(M+x)=\operatorname{out}(M)+x$ for all $x \in X$.

3. $\operatorname{inn}(\lambda M)=\lambda \operatorname{inn}(M)$ for all $\lambda \in \mathbb{R} \backslash\{0\}$.

4. $\operatorname{out}(\lambda M)=\lambda \operatorname{out}(M)$ for all $\lambda \in \mathbb{R} \backslash\{0\}$.

The previous proposition is based on the trivial fact that $\operatorname{shl}(M+x, S+x)=\operatorname{shl}(M, S)+x$ for all $x \in X$ and $\operatorname{shl}(\lambda M, \lambda S)=\lambda \operatorname{shl}(M, S)$ for all $\lambda \in \mathbb{R} \backslash\{0\}$. In general, if $T: X \rightarrow Y$ is an isomorphism of vector spaces, then $T(\operatorname{shl}(M, S))=\operatorname{shl}(T(M), T(S))$ for every nonempty subset $M$ of $X$ and every subset $S$ of $M$. We will explore next whether or not other related maps preserve this structure.

Recall that a map $f: X \rightarrow Y$ between vector spaces $X$ and $Y$ is said to be affine provided that $f(t x+(1-t) y)=t f(x)+(1-t) f(y)$ for all $x, y \in X$ and all $t \in \mathbb{R}$.

Theorem 1. Let $X$ and $Y$ be vector spaces. If $M$ is a convex subset of $X$ and $f: X \rightarrow Y$ is an affine map such that $f(M)$ is not a singleton, then $f(\operatorname{inn}(M)) \subseteq \operatorname{inn}(f(M))$.

Proof. Let $x \in \operatorname{inn}(M)$. We will show that $f(x) \in \operatorname{inn}(f(M))$. In first place, since $M$ is convex and $f$ is affine, $f(M)$ is also convex. By hypothesis, since $f(M)$ is not a singleton. Now, we can make use of Remark 1. So let $z \in f(M) \backslash\{f(x)\}$. There exists $y \in M \backslash\{x\}$ with $f(y)=z$. Since $x \in \operatorname{inn}(M)$, by Remark 1 , there exists $t<0$ such that $t x+(1-$ $t) y \in M$. Then $t f(x)+(1-t) f(y)=f(t x+(1-t) y) \in f(M)$. In other words, $f(x) \in$ $(f(y), f(t x+(1-t) y))$. This shows that $f(x) \in \operatorname{inn}(f(M))$.

The hypothesis that $f(M)$ is not a singleton cannot be removed. Indeed, any constant function is trivially affine and a constant function cannot verify the thesis of the previous theorem. The following example also shows that the hypothesis that $f(M)$ is not a singleton cannot be removed from Theorem 1.

Example 1. Let $X$ be a vector space of dimension strictly greater than 1 . Let $Y$ be a proper subspace of $X$ and let $p: X \rightarrow Y$ be a linear projection from $X$ onto $Y$. Notice that $p$ is affine and $\operatorname{inn}(\operatorname{ker}(p))=\operatorname{ker}(p)$ in view of [1] (Theorem 2.1). However, $p(\operatorname{inn}(\operatorname{ker}(p)))=p(\operatorname{ker}(p))=$ $\{0\}$ and so by [1] (Definition 1.2) we have that $\operatorname{inn}(p(\operatorname{ker}(p)))=\varnothing$.

The next result shows that the reverse inclusion $\operatorname{inn}(f(M)) \subseteq f(\operatorname{inn}(M))$ does not hold.

Theorem 2. Let $X$ be an infinite dimensional vector space. There exists a convex subset $M$ of $X$ and an affine map $f: X \rightarrow X$ such that $\operatorname{inn}(M)=f(\operatorname{inn}(M))=\varnothing$ and $\operatorname{inn}(f(M)) \neq \varnothing$.

Proof. In accordance with [1] (Corollary 5.3), there exists a non-singleton convex subset $M$ of $X$ free of inner points. It is clear then that $f(\operatorname{inn}(M))=\varnothing$ for any map $f: X \rightarrow X$. By making a translation if necessary, in virtue of Proposition 3(1) we may assume without any loss of generality that $0 \in M$. Fix an arbitrary non-zero element $m_{0} \in M$. Let $g: X \rightarrow \mathbb{R}$ be a linear functional with $g\left(m_{0}\right)=1$ and define $f: X \rightarrow \mathbb{R} m_{0} \subseteq X$ by $f(x)=g(x) m_{0}$ for all $x \in X$. Please note that $f$ is linear, so it is affine. Finally, $f(M)$ is a non-singleton convex subset of the finite dimensional vector space $\mathbb{R} m_{0}$, therefore $\operatorname{inn}(f(M)) \neq \varnothing$ in virtue of [1] (Theorem 5.1).

Recall that a function $f: X \rightarrow \mathbb{R}$ is said to convex, where $X$ is a vector space, if $f(t x+(1-t) y) \leq t f(x)+(1-t) f(y)$ for all $x, y \in X$ and all $t \in[0,1]$. In general, convex maps do not preserve inner structure. 
A metrizable topological vector space can always be endowed with a metric invariant under translations, i.e., $d(x+z, y+z)=d(x, y)$ for all $x, y, z$ (see [16,17]). If this metric is also sub-homogeneous, i.e., $d(t x, 0) \leq|t| d(x, 0)$, then we call it a sub-homogeneous metrizable topological vector space.

Lemma 1. In a metric space $X$, the distance function

$$
\begin{aligned}
d(\bullet, A): \quad X & \rightarrow \mathbb{R} \\
x & \mapsto d(x, A)
\end{aligned}
$$

is always uniformly continuous, where $A$ is a nonempty subset of X. In fact, $|d(x, A)-d(y, A)| \leq$ $d(x, y)$ for all $x, y \in X$.

Proof. Let $x, y \in X$. Fix an arbitrary $\varepsilon>0$ and let $a \in A$ such that $d(y, a)-d(y, A) \leq \varepsilon$. Then

$$
\begin{aligned}
d(x, A)-d(y, A) & \leq d(x, a)-d(y, a)+d(y, a)-d(y, A) \\
& \leq d(x, y)+d(y, a)-d(y, a)+d(y, a)-d(y, A) \\
& \leq d(x, y)+\varepsilon
\end{aligned}
$$

The arbitrariness of $\varepsilon$ assures that $d(x, A)-d(y, A) \leq d(x, y)$. In a similar way, it can be shown that $d(y, A)-d(x, A) \leq d(x, y)$.

Lemma 2. Let $X$ be a sub-homogeneous metrizable topological vector space. If $A$ is a convex subset of $X$, then the map

$$
\begin{aligned}
d(\bullet, A): \quad X & \rightarrow \mathbb{R} \\
x & \mapsto d(x, A)
\end{aligned}
$$

is convex.

Proof. Let $d$ be a translation-invariant sub-homogeneous metric on $X$ generating the topology of $X$. Let $x, y \in X$ and $t \in(0,1)$. Fix an arbitrary $\varepsilon>0$. We can find $a, b \in A$ such that $d(x, a)<d(x, A)+\varepsilon$ and $d(y, b)<d(y, A)+\varepsilon$. Then

$$
\begin{aligned}
d(t x+(1-t) y, A) & \leq d(t x+(1-t) y, t a+(1-t) b) \\
& =d(t x+(1-t) y-t a-(1-t) b, 0) \\
& =d(t(x-a)+(1-t)(y-b), 0) \\
& =d(t(x-a),-(1-t)(y-b)) \\
& \leq d(t(x-a), 0)+d(0,-(1-t)(y-b)) \\
& =d(t(x-a), 0)+d((1-t)(y-b), 0) \\
& \leq t d(x-a, 0)+(1-t) d(y-b, 0) \\
& =t d(x, a)+(1-t) d(y, b) \\
& <t d(x, A)+t \varepsilon+(1-t) d(y, A)+(1-t) \varepsilon \\
& =t d(x, A)+(1-t) d(y, A)+\varepsilon .
\end{aligned}
$$

The arbitrariness of $\varepsilon>0$ implies that $d(t x+(1-t) y, A) \leq t d(x, A)+(1-t) d(y, A)$.

Theorem 3. Let $X$ be a sub-homogeneous metrizable topological vector space. Let $A$ be a convex subset of $X$ with $\operatorname{inn}(A) \neq \varnothing$. Fix $a_{0} \in \operatorname{inn}(A)$. Then the function

$$
\begin{aligned}
f: X & \rightarrow \mathbb{R} \\
x & \mapsto f(x):=d\left(x, a_{0}\right)
\end{aligned}
$$

is convex, $f(A)$ is also convex, but $f(\operatorname{inn}(A)) \nsubseteq \operatorname{inn}(f(A))$. 
Proof. In view of Lemma 2, we have that $f$ is convex. Since $f$ is continuous (see Lemma 1 ) and $A$ is connected, we conclude that $f(A)$ is also connected and thus convex. Now observe that $a_{0} \in \operatorname{inn}(A)$ and $f\left(a_{0}\right)=0 \notin \operatorname{inn}(f(A))$ since $f(A) \subseteq[0, \infty)$.

The following example also serves to show that the inclusion $f(\operatorname{inn}(M)) \subseteq \operatorname{inn}(f(M))$ does not hold in general for convex functions.

Example 2. Let $X$ be a vector space of $\operatorname{dim}(X)>1$. Let $Y$ be a proper subspace of $X$ and $p: X \rightarrow X$ a linear projection of range $Y$. Fix $x_{0} \in \operatorname{ker}(p) \backslash\{0\}$. Take $M:=\left[0, x_{0}\right]$ and bear in mind that $\operatorname{inn}\left(\left[0, x_{0}\right]\right)=\left(0, x_{0}\right)$. Let $g: X \rightarrow \mathbb{R}$ be a linear functional with $g\left(x_{0}\right)=1$. Observe that $f:=g \circ p$ is a convex map. Now, $f(M)=\{0\}$ and thus $\operatorname{inn}(f(M))=\varnothing$. Finally, $f(\operatorname{inn}(M))=f\left(\left(0, x_{0}\right)\right)=\{0\}$.

The final result in this section highlights that the reverse inclusion $\operatorname{inn}(f(M)) \subseteq$ $f(\operatorname{inn}(M))$ does not hold either for convex functions.

Theorem 4. Let $X$ be an infinite dimensional vector space. There exists a convex subset $M$ of $X$ and a convex map $f: X \rightarrow \mathbb{R}$ such that $\operatorname{inn}(M)=f(\operatorname{inn}(M))=\varnothing$ and $\operatorname{inn}(f(M)) \neq \varnothing$.

Proof. In accordance with [1] (Corollary 5.3), there exists a non-singleton convex subset $M$ of $X$ free of inner points. It is clear then that $f(\operatorname{inn}(M))=\varnothing$ for any map $f: X \rightarrow \mathbb{R}$. By making a translation if necessary, in virtue of Proposition 3(1) we may assume without any loss of generality that $0 \in M$. Fix an arbitrary non-zero element $m_{0} \in M$. Let $f: X \rightarrow \mathbb{R}$ be a linear functional with $f\left(m_{0}\right)=1$. Please note that $f$ is linear, so it is convex. Finally, $f(M)$ is a non-singleton convex subset of the finite dimensional vector space $\mathbb{R}$, therefore $\operatorname{inn}(f(M)) \neq \varnothing$ in virtue of [1] (Theorem 5.1).

\section{Revisiting the Finest Locally Convex Vector Topology from the Inner Structure Viewpoint}

An affine manifold in a vector space $X$ is a translation of a vector subspace of $X$, i.e., $x+Y$ where $x \in X$ and $Y$ is a vector subspace of $X$. Observe that an affine manifold $x+Y$ contains 0 if and only if $x+Y=Y$.

Proposition 4. Let $X$ be a vector space. If $\left(x_{i}+Y_{i}\right)_{i \in I}$ is a family of affine manifolds of $X$ such that $\bigcap_{i \in I}\left(x_{i}+Y_{i}\right) \neq \varnothing$, then

$$
\bigcap_{i \in I}\left(x_{i}+Y_{i}\right)=x+\operatorname{span}\left(\bigcap_{i \in I}\left(x_{i}+Y_{i}\right)-x\right)
$$

for every $x \in \bigcap_{i \in I}\left(x_{i}+Y_{i}\right)$. In other words, $\bigcap_{i \in I}\left(x_{i}+Y_{i}\right)$ is an affine manifold of $X$.

Proof. It is cleat that

$$
\bigcap_{i \in I}\left(x_{i}+Y_{i}\right) \subseteq x+\operatorname{span}\left(\bigcap_{i \in I}\left(x_{i}+Y_{i}\right)-x\right)
$$

for every $x \in \bigcap_{i \in I}\left(x_{i}+Y_{i}\right)$. A generic element of $x+\operatorname{span}\left(\bigcap_{i \in I}\left(x_{i}+Y_{i}\right)-x\right)$ has the form $x+\sum_{j=1}^{k} \lambda_{j}\left(b_{j}-x\right)$, where $b_{j} \in \bigcap_{i \in I}\left(x_{i}+Y_{i}\right)$ and $\lambda_{j} \in \mathbb{R}$ for all $j \in\{1, \ldots, k\}$. Fix an arbitrary $i_{0} \in I$. Then $b_{j}=x_{i_{0}}+y_{i_{0} j}$, with $y_{i_{0} j} \in Y_{i_{0}}$ for all $j \in\{1, \ldots, k\}$. Observe that $x=x_{i_{0}}+y_{i_{0}}$ for some $y_{i_{0}} \in Y_{i_{0}}$. Then

$$
x+\sum_{j=1}^{k} \lambda_{j}\left(b_{j}-x\right)=x_{i_{0}}+y_{i_{0}}+\sum_{j=1}^{k} \lambda_{j}\left(y_{i_{0} j}-y_{i_{0}}\right) \in x_{i_{0}}+Y_{i_{0}} .
$$


The arbitrariness of $i_{0}$ implies that

$$
x+\operatorname{span}\left(\bigcap_{i \in I}\left(x_{i}+Y_{i}\right)-m\right) \subseteq \bigcap_{i \in I}\left(x_{i}+Y_{i}\right) .
$$

The affine hull of a subset $M$ of a vector space $X$ is defined as the intersection of all affine manifolds of $X$ containing $M$. The affine hull of $M$ is denoted by aff $(M)$. Since $\operatorname{span}(M)$ is trivially an affine manifold of $X$ containing $M$, then it is clear that aff $(M) \subseteq$ $\operatorname{span}(M)$. The following technical lemma will be useful.

Lemma 3. Let $X$ be a vector space and $M$ a non-empty subset of $X$. Then:

1. $\operatorname{aff}(M)=m+\operatorname{span}(M-m)$ for every $m \in M$.

2. $\operatorname{aff}(M)=\operatorname{span}(M)$ if and only if $0 \in \operatorname{aff}(M)$.

\section{Proof.}

1. Fix an arbitrary $m_{0} \in M$. By definition, $\operatorname{aff}(M)$ is the intersection of all affine manifolds containing $M$, therefore $\operatorname{aff}(M) \subseteq m_{0}+\operatorname{span}\left(M-m_{0}\right)$. Now let $V+x$ be an affine manifold of $X$ containing $M$, where $V$ is a vector subspace of $X$ and $x \in X$. Take an element $m_{0}+\lambda_{1}\left(m_{1}-m_{0}\right)+\cdots+\lambda_{k}\left(m_{k}-m_{0}\right) \in m_{0}+\operatorname{span}\left(M-m_{0}\right)$, where $m_{1}, \ldots, m_{k} \in M$. For every $i \in\{0,1, \ldots, k\}$ there exists $v_{i} \in V$ with $m_{i}=v_{i}+x$. Then $m_{0}+\lambda_{1}\left(m_{1}-m_{0}\right)+\cdots+\lambda_{k}\left(m_{k}-m_{0}\right)=\left(v_{0}+\lambda_{1}\left(v_{1}-v_{0}\right)+\cdots+\lambda_{k}\left(v_{k}-v_{0}\right)\right)+$ $x \in V+x$. This shows that $m_{0}+\operatorname{span}\left(M-m_{0}\right) \subseteq V+x$ and hence $m_{0}+\operatorname{span}(M-$ $\left.m_{0}\right) \subseteq \operatorname{aff}(M)$.

2. If $\operatorname{aff}(M)=\operatorname{span}(M)$, then $0 \in \operatorname{span}(M)=\operatorname{aff}(M)$. Conversely, assume that $0 \in$ $\operatorname{aff}(M)$. By the previous item, if we choose any $m_{0} \in M$, then we have that $0 \in$ $m_{0}+\operatorname{span}\left(M-m_{0}\right)$, which implies that $-m_{0} \in \operatorname{span}\left(M-m_{0}\right)$ and so $m_{0}+\operatorname{span}(M-$ $\left.m_{0}\right)=\operatorname{span}\left(M-m_{0}\right)$. Then $\operatorname{aff}(M)=\operatorname{span}\left(M-m_{0}\right)$ so the affine hull of $M$ is a vector subspace of $X$, which then implies that $\operatorname{span}(M) \subseteq \operatorname{aff}(M)$. Finally, we know that $\operatorname{aff}(M) \subseteq \operatorname{span}(M)$.

A point $a$ is said to be an internal point of a subset $A$ of a vector space $X$ provided that every line of $X$ passing through $a$ has a small interval around $a$ entirely contained in $A$, i.e., for every $x \in X \backslash\{a\}$ there exists $\varepsilon_{x}>0$ such that $a+\left(-\varepsilon_{x}, \varepsilon_{x}\right)(x-a) \subseteq A$. The set of internal points of $A$ is denoted by $\operatorname{inter}(A)$. On the other hand, $a$ is said to be an affine internal point of $A$ provided that every line of $\operatorname{aff}(A)$ passing through $a$ has a small interval around $a$ entirely contained in $A$, i.e., for every $x \in \operatorname{aff}(A) \backslash\{a\}$ there exists $\varepsilon_{x}>0$ such that $a+\left(-\varepsilon_{x}, \varepsilon_{x}\right)(x-a) \subseteq A$. Here we convey to assume that if $A$ is a singleton, then $\operatorname{aff}(A)=\varnothing$. The set of affine internal points of $A$ is denoted by ainter $(A)$.

Next, we recall the following useful and technical lemma, which is well known in the classical literature of topological vector spaces.

Lemma 4. Let $X$ be a topological vector space. Let $A$ be a convex subset of $X$. If $a \in \operatorname{int}(A)$ and $b \in \operatorname{cl}(A)$, then $[a, b) \subseteq \operatorname{int}(A)$. In particular, if $\operatorname{int}(A) \neq \varnothing$, then $\operatorname{int}(A)=\operatorname{int}(\operatorname{cl}(A))$ and $\mathrm{cl}(\operatorname{int}(A))=\mathrm{cl}(A)$, so $\operatorname{int}(A)$ is regular open and $\operatorname{cl}(A)$ is regular closed. If, in addition, $A$ is absolutely convex and $\operatorname{int}(A) \neq \varnothing$, then $0 \in \operatorname{int}(A)$.

It is very obvious that the the previous lemma does not hold if $A$ is not convex. The next lemma is crucial towards accomplishing the main results of this section. Very few items of the next lemma can be found in $[1,3]$, which we include here just for the sake of completeness. The rest are original. 
Lemma 5. Let $X$ be a topological vector space and $A$ a non-empty subset of $X$. Then:

1. $\quad \operatorname{int}(A) \subseteq \operatorname{inter}(A) \subseteq \operatorname{ainter}(A) \subseteq \operatorname{inn}(A) \subseteq A$.

2. If $A$ is open, then $A=\operatorname{int}(A)=\operatorname{inter}(A)=\operatorname{ainter}(A)=\operatorname{inn}(A)$.

3. If inter $(A) \neq \varnothing$, then $\operatorname{aff}(A)=M$ and $\operatorname{inter}(A)=\operatorname{ainter}(A)$.

4. If $A$ is convex, then $\operatorname{ainter}(A)=\operatorname{inn}(A)$.

5. If $A$ is convex and inter $(A) \neq \varnothing$, then $\operatorname{inter}(A)=\operatorname{ainter}(A)=\operatorname{inn}(A)$.

6. If $A$ is convex and $\operatorname{int}(A) \neq \varnothing$, then $\operatorname{int}(A)=\operatorname{inter}(A)=\operatorname{ainter}(A)=\operatorname{inn}(A)$.

7. If $A$ is convex, $\operatorname{int}(A) \neq \varnothing$ and $A=\operatorname{inter}(A)$, then $A$ is open.

\section{Proof.}

1. In view of [3] (Corollary 2.4), we have that $\operatorname{inter}(A) \subseteq \operatorname{ainter}(A) \subseteq \operatorname{inn}(A) \subseteq A$. We will show that $\operatorname{int}(A) \subseteq \operatorname{inter}(A)$. Fix an arbitrary $a \in \operatorname{int}(A)$. Let $x \in X \backslash\{a\}$. Let $V$ be a balanced and absorbing neighborhood of 0 such that $a+V \subseteq A$. Since $V$ is absorbing, we can find $\lambda>0$ such that $[-\lambda(x-a), \lambda(x-a)] \subseteq V$. Then $a+$ $[-\lambda, \lambda](x-a) \subseteq a+V \subseteq A$, which means that $a \in \operatorname{inter}(A)$.

2. If $A$ is open, then by (1), $A=\operatorname{int}(A) \subseteq \operatorname{inter}(A) \subseteq \operatorname{ainter}(A) \subseteq \operatorname{inn}(A) \subseteq A$.

3. Fix $a \in \operatorname{inter}(A)$. If $m \in M \backslash\{a\}$, then there exists a neighborhood $V$ of 0 in $\mathbb{R}$ such that $a+V(m-a) \subseteq A$. Take any $u \in V \backslash\{0\}$. Then $a+u(m-a) \in A$ so take $b \in A$ with $a+u(m-a)=b$. Please note that $m=a+u^{-1}(b-a) \in a+\operatorname{span}(A-a)=\operatorname{aff}(A)$ (see Lemma 3(1)).

4. This is [3] (Corollary 2.4).

5. This is [1] (Theorem 4.1).

6. Fix $a \in \operatorname{int}(A)$. Let $b \in \operatorname{inn}(A) \backslash\{a\}$. By Remark 1 , there exists $\lambda<0$ such that $\lambda a+(1-\lambda) b \in A$. In view of Lemma $4,[a, \lambda a+(1-\lambda) b) \subseteq \operatorname{int}(A)$. However, $b \in[a, \lambda a+(1-\lambda) b)$. As a consequence, $\operatorname{inn}(A) \subseteq \operatorname{int}(A)$ and by $(1), \operatorname{int}(A)=$ $\operatorname{inter}(A)=\operatorname{ainter}(A)=\operatorname{inn}(A)$.

7. $\quad$ By (5), $A=\operatorname{int}(A)=\operatorname{inter}(A)=\operatorname{ainter}(A)=\operatorname{inn}(A)$.

In [3] (Theorem 4.2), the finest locally convex vector topology is described in terms of the inner points:

Theorem 5. If $X$ is a vector space, then the set

$$
\{\operatorname{inn}(M): M \text { is convex, balanced and absorbing }\}
$$

is an open basis of neighborhoods of 0 for a locally convex vector topology $\tau_{X}$ on $X$ in such a way that if $\tau$ is another locally convex vector topology on $X$, then $\tau \subseteq \tau_{X}$.

Recall that a subset of vector space is absorbing if and only if 0 is an internal point of the set. The following result, which can be probably found on the classical literature of topological vector spaces, is proved here with our inner structure technique, for the sake of completeness.

Theorem 6. Let $X$ be a vector space. Let $A$ be a convex and absorbing subset of $X$. Then $A$ is $a$ neighborhood of 0 in the finest locally convex vector topology of X. More generally, if $A$ is convex and $\operatorname{inter}(A) \neq \varnothing$, then inter $(A)$ coincides with the interior of $A$ in the finest locally convex vector topology of $X$.

Proof. Let $\mathcal{L}:=\{B \subseteq A: B$ is absolutely convex $\}$. Clearly $\mathcal{L}$ is non-empty because $A$ is absorbing. Notice that $\mathcal{L}$ can be partially ordered by the inclusion. If $\left(B_{i}\right)_{i \in I}$ is a chain of $\mathcal{L}$, then it is clear that $\bigcup_{i \in I} B_{i} \in \mathcal{L}$. Using Zorn's Lemma, there exists a maximal element $B_{0}$. If $B_{0}$ is not absorbing, then there exists $x \neq 0$ and $\lambda>0$ such that $[-\lambda x, \lambda x] \subseteq A$ but $[-\lambda x, \lambda x] \nsubseteq B_{0}$. Then $C:=\operatorname{co}\left([-\lambda x, \lambda x] \cup B_{0}\right) \subseteq A$ is absolutely convex and contains $B_{0}$ strictly. This is a contradiction, so $B_{0}$ is absorbing. Now, $B_{0}$ is absolutely convex and 
absorbing, so $\operatorname{inn}\left(B_{0}\right)$ is a neighborhood of 0 in the finest locally convex vector topology of $X$ in virtue of Theorem 5 . Thus, finally $\operatorname{inn}\left(B_{0}\right) \subseteq B_{0} \subseteq A$ is a neighborhood of 0 in the finest locally convex vector topology of $X$.

Suppose now that $A$ is convex and $\operatorname{inter}(A) \neq \varnothing$. By Lemma 5(1) we know that $\operatorname{int}(A) \subseteq \operatorname{inter}(A)$. Fix an arbitrary $a \in \operatorname{inter}(A)$. Now, $0 \in \operatorname{inter}(A-a)$ so $A-a$ is absorbing, and it is also convex, therefore we have proved that $A-a$ is a neighborhood of 0 in the finest locally convex topology of $X$. As a consequence, $a$ is an interior point of $A$ in the finest locally convex topology of $X$.

Corollary 1. Let $X$ be a vector space. If $A$ is convex subset of $X$ such that $A=\operatorname{inter}(A)$, then $A$ is open in the finest locally convex vector topology of $X$.

Proof. In the first place, by making a translation if necessary, we may assume that $0 \in A$ without any loss of generality. Then 0 is an internal point $A$, which is equivalent to the fact that $A$ is absorbing. According to Theorem 6, $A$ is a neighborhood of 0 in the finest locally convex vector topology of $X$. In view of Lemma 5(6), $A$ is open in the finest locally convex vector topology of $X$.

The following corollary generalizes Lemma 4. Observe that in view of Lemma 5(1), $\operatorname{out}(A) \subseteq \operatorname{cl}(A)$.

Corollary 2. Let $X$ be a vector space. Let $A$ be a convex subset of $X$. If $a \in \operatorname{inn}(A)$ and $b \in \operatorname{out}(A)$, then $[a, b) \subseteq \operatorname{inn}(A)$. If, in addition, $A$ is absolutely convex and $\operatorname{inter}(A) \neq \varnothing$, then $0 \in \operatorname{inter}(A)$.

Proof. In the first place, by making a translation if necessary, we may assume without any loss of generality that $a=0$. In view of Lemma 5(3), $\operatorname{inn}(A)=\operatorname{ainter}(A)$. Since $0 \in A$, by applying Lemma 3 we conclude that $\operatorname{aff}(A)=\operatorname{span}(A)$. As a consequence, in $Y:=\operatorname{span}(A)$ we have that $\operatorname{ainter}(A)=\operatorname{inter}(A)$. Thus, in $Y, 0 \in \operatorname{inn}(A)=\operatorname{inter}(A)$ and so $A$ is absorbing in $Y$. Now we call on Theorem 6 to conclude that $A$ is a neighborhood of 0 in the finest locally convex topology of $Y$. Next, since $0 \in \operatorname{int}_{Y}(A)$ and $b \in \operatorname{cl}_{Y}(A)$, where $\operatorname{int}_{Y}(A)$ and $\operatorname{cl}_{Y}(A)$ refer to the interior of $A$ and the closure of $A$, respectively, in the finest locally convex topology of $Y$, we can apply Lemma 4 to conclude that $[0, b) \subseteq \operatorname{int}_{Y}(A)$. Finally, we simply apply Lemma $5(4)$ to deduce that $[0, b) \subseteq \operatorname{inn}(A)$.

Let us assume now that $A$ is absolutely convex and $\operatorname{inter}(A) \neq \varnothing$. In view of Theorem 6, the interior of $A$ in the finest locally convex topology of $X$ coincides with $\operatorname{inter}(A)$, so it is non-empty and then by Lemma 4 we have that 0 is an interior point of $A$ in the finest locally convex topology of $X$, so $0 \in \operatorname{inter}(A)$.

\section{Linearly Open Sets}

This section is devoted to showing that Corollary 1 does not hold true if $A$ is not convex. First, we will introduce several definitions for simplification purposes.

Definition 2. Let $X$ be a vector space. A subset $A$ of $X$ is said to be

- linearly open if $A=\operatorname{inter}(A)$.

- linearly closed if $X \backslash A$ is linearly open.

- linearly bounded if $X$ contains no rays or lines.

Please observe that, by bearing in mind Lemma 5(2), open sets in any topological vector space are always linearly open, and in view of Corollary 1 , convex linearly open sets are always open in the finest locally convex vector topology.

In $[18,19]$ a different definition of linearly closed was introduced, which states that a subset of $A$ of vector space $X$ is linearly closed if every line of $X$ through a point of $A$ intersects $A$ in a line, a closed ray, or a closed segment. This definition was conceived for 
convex sets. We will show next that our definition of linearly closed is equivalent to this one of $[18,19]$ under the assumption of convexity.

Proposition 5. Let $X$ be a vector space. If $A \subseteq X$ is linearly closed in the sense of [18,19], then $A$ is linearly closed. Conversely, if $A$ is convex and linearly closed, then $A$ is linearly closed in the sense of $[18,19]$.

Proof. Assume first that $A$ is linearly closed in the sense of $[18,19]$. We will show that $X \backslash A=\operatorname{inter}(X \backslash A)$. Fix an arbitrary $x \in X \backslash A$. Suppose on the contrary that there exists a line $L$ passing through $x$ that does not have an interval around $x$ entirely contained in $X \backslash A$. Then we can find a sequence $\left(z_{n}\right)_{n \in \mathbb{N}}$ in $L \cap A$ converging to $x$. By hypothesis, $L \cap A$ is either a closed segment or a closed ray. Since $\left(z_{n}\right)_{n \in \mathbb{N}} \subseteq L \cap A$ and is convergent to $x$, we conclude that $x$ is in the closed segment or the closed ray, which contradicts the fact that $x \in X \backslash A$.

Conversely, assume that $A$ is convex and linearly closed. Let $L$ be a line intersecting $A$. Since $A$ is convex, $L \cap A$ is either a line, a ray or a segment. Suppose on the contrary that $L \cap A$ is, for instance, an open ray starting at some $x \in X \backslash A$. Since $x$ is an internal point of $X \backslash A, L$ has a small interval around $x$ entirely contained in $X \backslash A$. This is a contradiction with the fact that the open ray is contained in $A$. If $L \cap A$ is an open or semi-open segment, then a similar proof applies.

Recall that the linear closure of a set $A$ is defined as $\operatorname{lcl}(A):=\operatorname{inn}(A) \cup \operatorname{out}(A)$.

Proposition 6. Let $X$ be a vector space. If $A \subseteq X$ is linearly closed, then $A=\operatorname{lcl}(A)$. Conversely, if $A$ is convex and $A=\operatorname{lcl}(A)$, then $A$ is linearly closed.

Proof. Suppose first that $A$ is linearly closed. By definition, $A \subseteq \operatorname{lcl}(A)$. Assume on the contrary that there exists $x \in \operatorname{lcl}(A) \backslash A$. Then $x$ is an extreme of a maximal segment $S$ of $A$. Take an element $s$ lying in the interior of $S$. Then the segment $[s, x) \subseteq A$. Now, the straight line passing through $s$ and $x$ does not have an interval around $x$ entirely contained in $X \backslash A$. This contradicts the fact that $X \backslash A=\operatorname{inter}(X \backslash A)$.

Conversely, suppose that $A$ is convex and $A=\operatorname{lcl}(A)$. Fix an arbitrary $x \in X \backslash A$. Assume on the contrary that there exists a line $L$ passing through $x$ that does not have an interval around $x$ entirely contained in $X \backslash A$. Then we can find a sequence $\left(z_{n}\right)_{n \in \mathbb{N}}$ in $L \cap A$ converging to $x$. Since $A$ is convex, there exists $n \in \mathbb{N}$ such that $\left[z_{n}, x\right) \subseteq A$. Then $x \in \operatorname{lcl}(A) \backslash A$, which is a contradiction.

By combining Propositions 5 and 6, we obtain the following corollary.

Corollary 3. Let $X$ be a vector space. The following conditions are equivalent for a convex subset A of $X$ :

1. A is linearly closed.

2. $\quad A$ is linearly closed in the sense of $[18,19]$.

3. $A=\operatorname{lcl}(A)$.

Next, we proceed to construct a balanced and linearly open set which has empty interior in the finest locally convex vector topology. We will rely on a series of remarks.

In [14] (Theorem 3.2) a balanced and absorbing subset with empty interior is constructed in every separable normed space $X$ of dimension greater than or equal to 2 . The form of this set is given by

$$
\left(\bigcup_{x \in \mathrm{S}_{X} \backslash\left\{ \pm x_{n}: n \in \mathbb{N}\right\}}[-1,1] x\right) \cup\left(\bigcup_{n \in \mathbb{N}}\left[\frac{-1}{n}, \frac{1}{n}\right] x_{n}\right),
$$


where $\left(x_{n}\right)_{n \in \mathbb{N}}$ is a dense sequence in the unit sphere of $X, \mathrm{~S}_{X}$, such that $x_{n} \neq \pm x_{m}$ for all $n \neq m$ (see [14] (Lemma 3.1)). The above set verifies also that it is dense in the unit ball of $X, B_{X}$. By relying on [14] (Theorem 3.2), in [15] (Theorem 1.1) a balanced and absorbing set with empty interior is constructed in any Hausdorff locally convex topological vector space.

Remark 2. Let $X$ be a Hausdorff locally convex topological vector space and let $W$ be the balanced and absorbing subset of $X$ described in [15] (Theorem 1.1). For every $x \in X$, let $W_{x}:=x+\lambda_{x} W$, where $0<\lambda_{x}<1$. Notice that $x \in \operatorname{inter}\left(W_{x}\right)$. We will construct a fractal-like set $A$ inductively:

- $A_{0}:=W$.

- $A_{1}:=\bigcup_{x \in A_{0}} W_{x}$.

- $A_{2}:=\bigcup_{x \in A_{1}} W_{x}$.

- $\quad$ and so on $A_{n}:=\bigcup_{x \in A_{n-1}} W_{x}$.

Then $A:=\bigcup_{n>0} A_{n}$. Next, we will assume that for every $n \in \mathbb{N}$ and every $x, y \in A_{n}$, $\lambda_{x}=\lambda_{y}$. Then we call it $\lambda_{n}$, i.e., $W_{x}=x+\lambda_{n} W$ for every $x \in A_{n}$. As a consequence, by relaying on addition of sets,

$$
A_{n}=\bigcup_{x \in A_{n-1}} W_{x}=\bigcup_{x \in A_{n-1}}\left(x+\lambda_{n} W\right)=A_{n-1}+\lambda_{n} W
$$

for every $n \in \mathbb{N}$. Finally, by recurrence,

$$
A_{n}=A_{n-1}+\lambda_{n} W=A_{n-2}+\lambda_{n-1} W+\lambda_{n} W=\cdots=W+\lambda_{1} W+\cdots+\lambda_{n-1} W+\lambda_{n} W,
$$

for every $n \in \mathbb{N}$.

Remark 3. Please note that if $A \subseteq B$, then inter $(A) \subseteq \operatorname{inter}(B)$.

Theorem 7. Let $X$ be a Hausdorff locally convex topological vector space. Let $W$ be a balanced and absorbing subset of $X$ with empty interior. The fractal-like set A constructed in Remark 2 is balanced, absorbing and linearly open.

Proof. In the first place, $W_{x}$ is balanced because $W$ is also balanced. Therefore, $A$ is balanced because it is a union of balanced sets. It is also clear that $A$ is absorbing because it contains the absorbing set $W$. Let us see now that $A$ is linearly open. Fix an arbitrary $a \in A$. There exists $n \in \mathbb{N}$ such that $a \in A_{n}$. Then there exists $x \in A_{n-1}$ such that $a \in W_{x}$. Observe that $A_{n+1}=\bigcup_{x \in A_{n}} W_{x}$. Now, $a \in \operatorname{inter}\left(W_{a}\right)$ in view of Remark 2 . On the other hand, since $a \in A_{n}, W_{a} \subseteq A_{n+1} \subseteq A$. By bearing in mind Remark 3 , we finally conclude that $a \in \operatorname{inter}\left(W_{a}\right) \subseteq \operatorname{inter}(A)$.

We will modify a bit Equation (1) to easily construct a non-trivial non-convex balanced, absorbing and linearly open set which is a feasible candidate to have empty interior in the finest locally convex vector topology. First, we need the following technical lemma.

Lemma 6. Let $X$ be a normed space. Let $x \in \mathrm{S}_{X}$ and $\left(x_{n}\right)_{n \in \mathbb{N}} \subseteq \mathrm{S}_{X}$. If there exists $\varepsilon>0$ such that the ball of center $x$ and radius $\varepsilon, B_{X}(x, \varepsilon)$, does not contain any element of $\left\{ \pm x_{n}: n \in \mathbb{N}\right\}$, then for every $\lambda \in(-1,1) \backslash\{0\}$ there exists $\tau>0$ such that $B_{X}(\lambda x, \tau) \cap\left\{\gamma x_{n}: \gamma \in(-1,1), n \in\right.$ $\mathbb{N}\}=\varnothing$.

Proof. Suppose on the contrary that such $\tau$ does not exist. Then we can find a subsequence $\left(\gamma_{k} x_{n_{k}}\right)_{k \in \mathbb{N}}$ converging to $\lambda x$. Then $\left|\gamma_{k}\right|=\left\|\gamma_{k} x_{n_{k}}\right\| \rightarrow\|\lambda x\|=|\lambda|$ as $k \rightarrow \infty$. By passing to a subsequence of $\left(\gamma_{k}\right)_{k \in \mathbb{N}}$ if necessary, we can assume that either $\left(\gamma_{k}\right)_{k \in \mathbb{N}}$ converges to $\lambda$ or to $-\lambda$. Then either $\left(\lambda x_{n}\right)_{k \in \mathbb{N}}$ or $\left(-\lambda x_{n}\right)_{k \in \mathbb{N}}$ converges to $\lambda x$, which implies the contradiction that either $\left(x_{n_{k}}\right)_{k \in \mathbb{N}}$ or $\left(-x_{n_{k}}\right)_{k \in \mathbb{N}}$ converges to $x$.

Here is another example of a non-trivial non-convex, balanced and absorbing linearly open set. 
Theorem 8. Let $X$ be an infinite dimensional vector space. There exists a subset $A \subseteq X$ such that $A$ is balanced, absorbing and linearly open but it is not convex.

Proof. In the first place, let $\left(e_{i}\right)_{i \in I}$ be a Hamel basis for $X$. Let us consider the norm on $X$ defined by $\|x\|=\left|\lambda_{1}\right|+\cdots+\left|\lambda_{n}\right|$, where $x=\lambda_{1} e_{i_{1}}+\cdots+\lambda_{n} e_{i_{n}}$ is the linear decomposition of $x$ in the Hamel basis $\left(e_{i}\right)_{i \in I}$. Fix any infinite sequence $\left(x_{n}:=e_{i_{n}}\right)_{n \in \mathbb{N}}$ of different elements of $\left(e_{i}\right)_{i \in I}$. Notice that $\left\|e_{n} \pm e_{m}\right\|=2$ for every $n \neq m$. Observe that for every $x \in \mathrm{S}_{X} \backslash\left\{ \pm x_{n}: n \in \mathbb{N}\right\}$ there exists $\varepsilon>0$ such that $\mathrm{B}_{X}(x, \varepsilon) \cap\left\{ \pm x_{n}: n \in \mathbb{N}\right\}=\varnothing$. Also, for every $n \in \mathbb{N}, \mathrm{B}_{X}\left(x_{n}, 1\right) \cap\left\{ \pm x_{m}: m \in \mathbb{N} \backslash\{n\}\right\}=\varnothing$.

Let us define now

$$
A:=\left(\bigcup_{x \in \mathrm{S}_{X} \backslash\left\{ \pm x_{n}: n \in \mathbb{N}\right\}}(-1,1) x\right) \cup\left(\bigcup_{n \in \mathbb{N}}\left(\frac{-1}{n}, \frac{1}{n}\right) x_{n}\right),
$$

where $\left(x_{n}\right)_{n \in \mathbb{N}}$ is a any convergent sequence in the unit sphere of $X, \mathrm{~S}_{X}$, to some $x_{0} \in \mathrm{S}_{X}$ and such that $x_{n} \neq \pm x_{m}$ for all $n \neq m$. We will show that $A$ verifies the desired properties:

- $A$ is balanced and absorbing by construction.

- $A$ is not a neighborhood of 0 . Indeed, if there exists $\varepsilon>0$ such that $\varepsilon \mathrm{B}_{X} \subseteq A$, then we can find $n \in \mathbb{N}$ such that $\frac{1}{n}<\varepsilon$, so $\frac{x_{n}}{n} \in \varepsilon \mathrm{B}_{X} \subseteq A$, which is not possible by construction of $A$.

- $\quad A$ is not convex. Indeed, fix arbitrary elements $n \neq m$ in $\mathbb{N}$ with $n>3$, and notice that

$$
\frac{1}{2} \frac{x_{n}+x_{m}}{3}+\frac{1}{2} \frac{x_{n}-x_{m}}{3}=\frac{x_{n}}{3} \notin A .
$$

- $\quad A=\operatorname{inter}(A)$. Indeed, by construction, $A$ is absorbing and thus $0 \in \operatorname{inter}(A)$. Let $a \in A \backslash\{0\}$. We will distinguish two cases:

* $\quad a=\lambda x$ with $x \in \mathrm{S}_{X} \backslash\left\{ \pm x_{n}: n \in \mathbb{N}\right\}$ and $\lambda \in(-1,1) \backslash\{0\}$. In view of Lemma 6, there exists $\tau>0$ such that $\mathrm{B}_{X}(\lambda x, \tau) \cap\left\{\gamma x_{n}: \gamma \in(-1,1), n \in \mathbb{N}\right\}=\varnothing$. This shows that $a \in \operatorname{int}(A) \subseteq \operatorname{inter}(A)$.

$\star \quad a=\lambda x_{n}$ for some $\left.n \in \mathbb{N}\right\}$ and some $\lambda \in\left(\frac{-1}{n}, \frac{1}{n}\right) \backslash\{0\}$. By using again Lemma 6, there exists $\tau>0$ such that $\mathrm{B}_{X}\left(\lambda x_{n}, \tau\right) \cap\left\{\gamma x_{m}: \gamma \in(-1,1), m \in \mathbb{N} \backslash\{n\}\right\}=\varnothing$. This shows that $a \in \operatorname{int}(A) \subseteq \operatorname{inter}(A)$.

Finally, we present a simple way to construct linearly open sets with empty interior in any infinite dimensional Banach space.

Theorem 9. Let $X$ be a topological vector space. If there exists a non-continuous linear functional $f: X \rightarrow \mathbb{R}$, then there exists a subset $A \subseteq X$ such that $A$ is balanced and linearly open but it has empty interior in $X$.

Proof. Since $f$ is not continuous, then $f \neq 0$. Take $A:=X \backslash \operatorname{ker}(f)$. We will show that $A$ satisfies the required properties:

- $A$ is clearly balanced.

- $A$ is linearly open. Take $x \in X \backslash \operatorname{ker}(f)$. Fix an arbitrary $y \in X \backslash\{x\}$. If $f(y)=f(x)$, then $x+t(y-x) \in X \backslash \operatorname{ker}(f)$ for all $t \in \mathbb{R}$. If $f(y) \neq f(x)$, then $x+t(y-x) \in$ $X \backslash \operatorname{ker}(f)$ for all $|t|<\frac{|f(x)|}{|f(y)-f(x)|}$. Thus, $x \in \operatorname{inter}(X \backslash \operatorname{ker}(f))$.

- $\quad A$ has empty interior in $X$. Indeed, $\operatorname{ker}(f)$ is a maximal subspace of $X$ so $\operatorname{cl}(\operatorname{ker}(f))$ is either $\operatorname{ker}(f)$ or $X$. Since $f$ is not continuous, $\operatorname{ker}(f)$ cannot be closed, therefore $X=\operatorname{cl}(\operatorname{ker}(f))$. This implies that $\operatorname{ker}(f)$ is dense in $X$ and thus $A$ has empty interior in $X$. 
In [20] there can be found examples of Hausdorff locally convex vector spaces in which all linear functional are continuous as well as a characterization of topological vector spaces with non-continuous linear functionals.

\section{Extremal Sets and Inner Points}

We refer the reader to [21-23] for a wide perspective on geometrical concepts related to extremal structure.

Consider a non-empty subset $M$ of a topological vector space $X$. A subset $C$ of $M$ is said to be extremal in $M$ provided that $C$ verifies the extremal condition with respect to $M$ : if $x, y \in M$ and $t \in(0,1)$ are so that $t x+(1-t) y \in C$, then $x, y \in C$. A convex extremal subset is called a face. In [2] (Lemma 2.1) it is proved that if a face of a convex set intersects the inner points, then the face coincides with the whole set. In the non-convex settings different things occur.

Theorem 10. Let $X$ be a topological vector space and $M$ a non-empty subset of $X$. If $E$ is an extremal subset of $M$ such that $E \cap \operatorname{ainter}(M) \neq \varnothing$, then $E=M$.

Proof. Suppose that there exists $e \in E \cap \operatorname{ainter}(M)$. Take $m \in M$. Since $m \in \operatorname{aff}(M)$, therefore there exists $0<\varepsilon<1$ such that $e+t(m-e) \in M$ for every $-\varepsilon \leq t \leq \varepsilon$. Now,

$$
e=\frac{\varepsilon}{1+\varepsilon} m+\frac{1}{1+\varepsilon}(e-\varepsilon(m-e))
$$

is a convex combination where $m$ and $e-\varepsilon(m-e)$ are both in $M$. Since $E$ is extremal in $M$, we conclude that $m \in E$.

The next example shows that in the settings of the previous theorem, the fact that $E \cap \operatorname{inn}(M) \neq \varnothing$ does not necessarily imply that $E=M$.

Example 3. Let $X$ be a vector space with $\operatorname{dim}(X) \geq 2$. Fix $x, y \in X$ linearly independent. Take $M:=[-x, x] \cup\{y\}$. Observe that $E:=[-x, x]$ is extremal in $M$ and $\operatorname{inn}(M)=(-x, x)$, so $E \cap \operatorname{inn}(M) \neq \varnothing$ but $E \neq M$.

An element $m$ of a non-empty subset $M$ of a vector space $X$ is said to be an extremal point if $\{m\}$ is an extremal subset of $M$, and $m$ is called an extreme point of $M$ provided that the following conditions holds: if $C$ is a segment of $M$ containing $m$, then $m$ is an extreme of $C$. The set of extreme points of $M$ is usually denoted by $\operatorname{ext}(M)$ and the set of extremal points is denoted by $\operatorname{extl}(M)$. Obviously, $\operatorname{extl}(M) \subseteq \operatorname{ext}(M)$, and if $M$ is convex, then $\operatorname{extl}(M)=\operatorname{ext}(M)$.

Remark 4. Let $X$ be a topological vector space. If $N \subseteq M \subseteq X$, then $\operatorname{ext}(M) \cap N \subseteq \operatorname{ext}(N)$ and $\operatorname{extl}(M) \cap N \subseteq \operatorname{extl}(N)$. As a consequence, $\operatorname{ext}(M \backslash\{m\})=\operatorname{ext}(M) \backslash\{m\}$ for all $m \in M$. It is also verified that $\operatorname{extl}(M) \backslash\{m\} \subseteq \operatorname{extl}(M \backslash\{m\})$. The equality in the last inclusion is not true in general.

Lemma 7. Let $X$ be a topological vector space. Let $M$ be a subset of $X$ and $S$ a subset of $M$. Then $\operatorname{ext}(M) \backslash S \subseteq \operatorname{ext}(M \backslash S)$ and $\operatorname{extl}(M) \backslash S \subseteq \operatorname{extl}(M \backslash S)$. If $S$ is convex and closed in $M$, then $\operatorname{ext}(M \backslash S)=\operatorname{ext}(M) \backslash S$.

Proof. In view of Remark 4, we have that $\operatorname{ext}(M) \backslash S \subseteq \operatorname{ext}(M \backslash S)$ and $\operatorname{extl}(M) \backslash S \subseteq$ $\operatorname{extl}(M \backslash S)$. Assume that $S$ is convex and closed in $M$. Fix an arbitrary $m \in \operatorname{ext}(M \backslash S)$ and suppose that $m \notin \operatorname{ext}(M)$. Take a closed segment $C$ of $M$ whose interior contains $m$. Since $S$ is convex and closed in $M$, we conclude that $C \cap S$ is a closed segment in $M$. Please note that $m \notin C \cap S$ and $C \backslash S$ is open in $C$. Therefore there exists an open segment $(c, d) \subseteq C \backslash S$ such that $m \in(c, d)$. Since $(c, d) \subseteq M \backslash S$, we reach the contradiction that $m \notin \operatorname{ext}(M \backslash S)$. 
Theorem 11. Let $X$ be a vector space. Let $M$ be a closed convex subset of $X$ such that int $(M) \neq$ $\varnothing$. Then $\operatorname{extl}(M) \cap \operatorname{bd}(M)=\operatorname{extl}(\operatorname{bd}(M))$ and $\operatorname{ext}(M) \cap \operatorname{bd}(M)=\operatorname{ext}(\operatorname{bd}(M))$. As $a$ consequence, $\operatorname{ext}(\operatorname{bd}(M))=\operatorname{extl}(\operatorname{bd}(M))$.

Proof. Let $x \in \operatorname{extl}(\operatorname{bd}(M))$. If $m, n \in M$ and $t \in(0,1)$ are so that $x=t m+(1-t) n$, then Lemma 4 assures that both $m$ and $n$ lie in $\operatorname{bd}(M)$, which implies that $m=n=x$. This shows that $x \in \operatorname{extl}(M)$. Now let $x \in \operatorname{ext}(\operatorname{bd}(M))$. If $S$ is a segment of $M$ containing $x$ in its interior, then by using again Lemma 4 , the whole of $S$ lies in $\operatorname{bd}(M)$, which implies that $x$ is an extreme of $S$. This shows that $x \in \operatorname{ext}(M)$. Finally, the convexity of $M$ allows that $\operatorname{extl}(\operatorname{bd}(M))=\operatorname{extl}(M) \cap \operatorname{bd}(M)=\operatorname{ext}(M) \cap \operatorname{bd}(M)=\operatorname{ext}(\operatorname{bd}(M))$.

\section{Convex Components and Inner Structure}

Recall that a convex component is simply a maximal convex subset. In [24] (Theorem 2.3) it is proved that if a subset of a topological vector space is closed, then so are all of its convex components. Here we will relate the inner points of the convex components with the inner points of the whole.

Convex components behave very differently from connected components. In fact, different convex components can have non-empty interior. If $C$ and $D$ are different convex components pf a set $M$ and $C \cap D \neq \varnothing$, then Zorn's Lemma guarantees the existence of a third convex component containing $C \cap D$.

On the other hand, notice that in general, if $C \subseteq D$, then it is not satisfied that $\operatorname{inn}(C) \subseteq \operatorname{inn}(D)$ nor $\operatorname{inn}(D) \cap C \subseteq \operatorname{inn}(C)$.

Theorem 12. Let $X$ be a vector space. Let $M$ a nonempty subset of $X$. If the convex components $\left\{C_{i}\right\}_{i \in I}$ of $M$ are pairwise disjoint, then $\operatorname{inn}(M)=\bigcup_{i \in I} \operatorname{inn}\left(C_{i}\right)$.

Proof. Let $x \in \operatorname{inn}(M)$ and let $i \in I$ such that $x \in C_{i}$. First, we need to prove that $\operatorname{shl}\left(C_{i}, x\right) \neq \varnothing$. Please note that if $C_{i}$ is a singleton, then $C_{i}=\{x\}$ and since all the convex components of $M$ are disjoint, then $\operatorname{shl}(M, x)=\varnothing$, which contradicts the fact that $x \notin \operatorname{inn}(M)$. Thus, $C$ is not a singleton and the convexity of $C_{i}$ allows that $\operatorname{shl}\left(C_{i}, x\right) \neq \varnothing$. Next, let $T$ be a maximal element of $C_{i}$ containing $x$. Again, since the convex components of $M$ are pairwise disjoint, $T$ has to be a maximal element of $\operatorname{shl}(M, x)$. Since $x \in \operatorname{inn}(M)$, we have that $x$ lies in the interior of $T$. This shows that $x \operatorname{inn}\left(C_{i}\right)$. Conversely, let $x \in$ $\bigcup_{i \in I} \operatorname{inn}\left(C_{i}\right)$ and fix $i \in I$ so that $x \in \operatorname{inn}\left(C_{i}\right)$. Notice that $\varnothing \neq \operatorname{shl}\left(C_{i}, x\right) \subseteq \operatorname{shl}(M, x)$. Take a maximal element $T$ of $\operatorname{shl}(M, x)$. There exists a convex component containing $T$. However, $T$ contains $x$ and the convex component containing $x$ is $C_{i}$, so the fact that all the convex components of $M$ are pairwise disjoint, implies that $T \subseteq C_{i}$. Then $T$ is a maximal element of $\operatorname{shl}\left(C_{i}, x\right)$. Since $x \in \operatorname{inn}\left(C_{i}\right)$, we conclude that that $x$ lies in the interior of $T$. This proves that $x \in \operatorname{inn}(M)$.

The following theorem assures that if a set is linearly closed, then so are its convex components. This theorem already appears in [24] (Corollary 2.5) but it was only proved for the case where the set is closed in the finest locally convex vector topology. Here we provide the full proof to finalize this manuscript.

Theorem 13. Let $X$ be a vector space. If $A \subseteq X$ is linearly closed, then the convex components of $A$ are also linearly closed.

Proof. Let $C$ be a convex component of $A$. We will show that $X \backslash C=\operatorname{inter}(X \backslash C)$. Suppose on the contrary that there exists $x \in(X \backslash C) \backslash \operatorname{inter}(X \backslash C)$. Then we can find a line $L$ of $X$ through $x$ which does not have an interval around $x$ entirely contained in $X \backslash C$. Then there exists a sequence $\left(z_{n}\right)_{n \in \mathbb{N}} \subseteq L \cap C$ which is convergent to $x$. Since $C$ is convex, there exists $n \in \mathbb{N}$ such that $\left[z_{n}, x\right) \subseteq C$. At this stage, observe that $x \in A$. Indeed, if $x \notin A$, then $x \in X \backslash A=\operatorname{inter}(X \backslash A)$, so $L$ must have an interval $S$ around $x$ entirely 
contained in $X \backslash A$. Then $S \subseteq X \backslash A \subseteq X \backslash C$. This contradicts our initial assumption that $x \in(X \backslash C) \backslash \operatorname{inter}(X \backslash C)$. As a consequence, $x \in A$. We will reach a contradiction by showing that $\operatorname{co}(C \cup\{x\}) \subseteq A$. Assume on the contrary that there exists $t \in(0,1)$ and $c \in C$ such that $t c+(1-t) x \in X \backslash A$. Consider the 3-simplex $\operatorname{co}\left(\left\{c, z_{n}, x\right\}\right)$. Since $c \in C$ and $\left[z_{n}, x\right) \subseteq C$ and $C$ is convex, we have that the segment $\left[c, z_{n}\right] \subseteq C$ and the interior of the 3-simplex is also contained in $C$. Finally, since $t c+(1-t) x \in X \backslash A=\operatorname{inter}(X \backslash A)$, the line passing through $t c+(1-t) x$ and $z_{n}$ must have a small interval around $t c+(1-t) x$ entirely contained in $X \backslash A \subseteq X \backslash C$. A portion of this interval lies in the interior of the 3-simplex $\operatorname{co}\left(\left\{c, z_{n}, x\right\}\right)$ which is contained in $C$. This is a contradiction. Thus, $\operatorname{co}(C \cup\{x\}) \subseteq A$ and this contradicts the fact that $C$ is a convex component of $A$. Therefore, $x \in \operatorname{inter}(X \backslash C)$ and hence $X \backslash C=\operatorname{inter}(X \backslash C)$.

Author Contributions: Conceptualization, F.J.G.-P., S.M.-P., E.N.-G. and A.S.-A.; Formal analysis, F.J.G.-P., S.M.-P., E.N.-G. and A.S.-A.; Investigation, F.J.G.-P., S.M.-P., E.N.-G. and A.S.-A.; Methodology, F.J.G.-P., S.M.-P., E.N.-G. and A.S.-A. All authors have read and agreed to the published version of the manuscript.

Funding: This research was funded by Ministry of Science, Innovation and Universities of Spain, grant number PGC-101514-B-I00; and by the 2014-2020 ERDF Operational Programme and by the Department of Economy, Knowledge, Business and University of the Regional Government of Andalusia, grant number FEDER-UCA18-105867.

Institutional Review Board Statement: Not applicable.

Informed Consent Statement: Not applicable.

Data Availability Statement: Not applicable.

Acknowledgments: The authors would like to dedicate this work to our dear colleague and friend Andrés Pastor. The authors would also like to thank Fulgencio Meseguer Galán for his financial support.

Conflicts of Interest: The authors declare no conflict of interest. The funders had no role in the design of the study; in the collection, analyses, or interpretation of data; in the writing of the manuscript, or in the decision to publish the results.

\section{References}

1. García-Pacheco, F.J.; Naranjo-Guerra, E. Inner structure in real vector spaces. Georgian Math. J. 2020, 27, 361-366. [CrossRef]

2. García-Pacheco, F.J. A Solution to the Faceless Problem. J. Geom. Anal. 2020, 30, 3859-3871. [CrossRef]

3. García-Pacheco, F.J. Relative interior and closure of the set of inner points. Quaest. Math. 2020, 43, 761-772. [CrossRef]

4. Bourbaki, N. Elements of Mathematics. Topological Vector Spaces; Chapters 1-5; Springer: Berlin, Germany, 1987 ; p. viii+364. [CrossRef]

5. Dubins, L.E. On extreme points of convex sets. J. Math. Anal. Appl. 1962, 5, 237-244. [CrossRef]

6. Kakutani, S.; Klee, V. The finite topology of a linear space. Arch. Math. 1963, 14, 55-58. [CrossRef]

7. Zelazko, W. The strongest vector space topology is locally convex on separable linear subspaces. Ann. Pol. Math. 1997, 66, 275-282. [CrossRef]

8. Diestel, J.; Morris, S.A.; Saxon, S.A. Varieties of locally convex topological vector spaces. Bull. Am. Math. Soc. 1971, 77, 799-803. [CrossRef]

9. Köthe, G. Topological Vector Spaces. I; Garling. Die Grundlehren der mathematischen Wissenschaften, Band 159; Springer: New York, NY, USA, 1969; p. xv+456.

10. Morris, S.A.; Diestel, J. Remarks on varieties of locally convex linear topological spaces. J. Lond. Math. Soc. 1974, 8, 271-278. [CrossRef]

11. García-Pacheco, F.J.; Pérez-Fernández, F.J. Pre-Schauder Bases in Topological Vector Spaces. Symmetry 2019, 11, 1026. [CrossRef]

12. Garcia-Pacheco, F.J. Regularity in Topological Modules. Mathematics 2020, 8, 1580. [CrossRef]

13. García-Pacheco, F.J. Convex functions on topological modules. Linear Multilinear Algebra 2020, 1-14. [CrossRef]

14. García-Pacheco, F.J.; Naranjo-Guerra, E. Balanced and absorbing subsets with empty interior. Adv. Geom. 2016, 16, 477-480. [CrossRef]

15. García-Pacheco, F.J.; Naranjo-Guerra, E. A family of balanced and absorbing sets with empty interior. J. Nonlinear Funct. Anal. 2017, 2017, 1-10. [CrossRef]

16. Rolewicz, S. Metric Linear Spaces, 2nd ed.; Volume 20 Mathematics and Its Applications (East European Series); D. Reidel Publishing Co.: Dordrecht, The Netherlands; PWN-Polish Scientific Publishers: Warsaw, Poland, 1985; p. xii+459. 
17. Kakutani, S. Über die Metrisation der topologischen Gruppen. Proc. Imp. Acad. Tokyo 1936, 12, 82-84. [CrossRef]

18. Fullerton, R.E. On a semi-group of subsets of a linear space. Proc. Am. Math. Soc. 1950, 1, 440-442. [CrossRef]

19. Fullerton, R.E. An intersection property for cones in a linear space. Proc. Am. Math. Soc. 1958, 9, 558-561. [CrossRef]

20. García-Pacheco, F.J. Non-continuous linear functionals on topological vector spaces. Banach J. Math. Anal. 2008, 2, 11-15. [CrossRef]

21. Aizpuru, A.; García-Pacheco, F.J. A short note about exposed points in real Banach spaces. Acta Math. Sci. Ser. B 2008, 28, 797-800. [CrossRef]

22. Diestel, J.; Uhl, J.J., Jr. Vector Measures; American Mathematical Society: Providence, RI, USA, $1977 ;$ p. xiii+322.

23. Diestel, J. Geometry of Banach Spaces—Selected Topics; Springer: Berlin, Germany; New York, NY, USA, $1975 ;$ p. xi+282.

24. García-Pacheco, F.J. Convex components and multi-slices in real topological vector spaces. Ann. Funct. Anal. 2015, 6, 73-86. [CrossRef] 